\title{
DECISIONES DE CONSUMO Y PORTAFOLIO BAJO CONDICIONES DE RIESGO E INCERTIDUMBRE
}

\author{
Francisco Venegas-Martinez* \\ Centro de Investigación en Finanzas \\ Tecnológico de Monterrey, Campus Ciudad de México
}

(Received 2 June 2005, accepted 15 December 2005)

\section{Resumen}

La presente investigación presenta un modelo que describe el comportamiento de un consumidor-inversionista, adverso al riesgo, que toma decisiones de consumo y portafolio en un ambiente de riesgo e incertidumbre. Con el propósito de generar soluciones analíticamente tratables del problema de maximización de utilidad de un consumidor racional, la estructura de la economía se mantiene lo más simple posible. Se supone que en la economía está poblada por agentes idénticos de vida infinita, los cuales obtienen satisfacción por el consumo de un bien de carácter perecedero.

\begin{abstract}
This investigation is aimed to develop a model that describes the behavior of a risk-averse consumer-investor that makes consumption and portfolio decisions under risk and uncertainty. With the purpose to generate an analytical solution of the utility maximization problem of a rational consumer, the structure of economy stays as simple as possible. It is assumed that the economy is populated by identical and infinitely lived agents, who obtain satisfaction from the consumption of a perishable good.
\end{abstract}

Classificación JEL: F41, F31, G13.

Palabras clave: Stochastic modeling.

* Tecnológico de Monterrey, Campus Ciudad de México, Centro de Investigación en Finanzas, Calle del Puente 222, 14380, México, D. F., Teléfono (52)55 54831861, Correo Electrónico: fvenegas@itesm.mx

The author wishes to thank two anonymous referees of this journal for valuable guidance and suggestions. 


\section{Introducción}

A continuación se desarrolla un modelo estocástico que describe el comportamiento de un agente adverso al riesgo que toma decisiones de consumo y portafolio. Se supone que en la economía los agentes obtienen satisfacción por el consumo de un bien y que tienen acceso a activos financieros sujetos al riesgo de mercado. Otras investigaciones relacionadas con el tema se encuentran en Venegas-Martínez (2000) y (2005).

La organización del presente trabajo es como sigue. En el transcurso de la. próxima sección se define la dinámica del nivel general de precios. En la sección 3 se describen los diferentes activos a que los consumidores tienen acceso. En el sección 4 se determinan los rendimientos de los activos. En la sección 5 se resuelve el problema de decisión de un agente representativo. En la sección 6 se presentan las conclusiones. Por último, un par de apéndices proporcionan detalles analíticos del problema de decisión.

\section{Dinåmica estocástica del nivel general de precios}

Se supone que los individuos perciben que el precio del bien, $P_{t}$, es conducido por un proceso estocástico de difusión con saltos:

$$
\mathrm{d} P_{t}=\pi P_{t} \mathrm{~d} t+\sigma_{P} P_{t} \mathrm{~d} W_{P, t}+\nu_{P} P_{t} \mathrm{~d} Q_{P, t},
$$

donde $\pi$ representa la tasa de inflación promedio esperada condicional a que ningún salto ocurra, $\sigma_{P}$ es la volatilidad esperada de la tasa de inflación y $1+\nu_{P}$ es el tamaño promedio esperado de posibles saltos en el nivel general de precios. El proceso $W_{P, t}$ es un proceso de Wiener estandarizado, es decir, $W_{P, t}$ presenta incrementos normales independientes con $\mathrm{E}\left[\mathrm{d} W_{P, t}\right]=0$ y $\operatorname{Var}\left[\mathrm{d} W_{P, t}\right]=\mathrm{d} t$. Se supone que los saltos en el nivel general de precios siguen un proceso de Poisson, $Q_{P, t}$, con parámetro de intensidad $\lambda_{P}$, de tal manera que

$$
\operatorname{Pr}\{\text { un salto unitario durante } \mathrm{d} t\}=\operatorname{Pr}\left\{\mathrm{d} Q_{P, t}=1\right\}=\lambda_{P} \mathrm{~d} t+o(\mathrm{~d} t),
$$

mientras que ${ }^{1}$

$$
\operatorname{Pr}\{\text { ningún salto en } \mathrm{d} t\}=\operatorname{Pr}\left\{\mathrm{d} Q_{P, t}=0\right\}=1-\lambda_{P} \mathrm{~d} t+o(\mathrm{~d} t) .
$$

Por lo tanto, $\mathrm{E}\left[\mathrm{d} Q_{P, t}\right]=\operatorname{Var}\left[\mathrm{d} Q_{P, t}\right]=\lambda_{P} \mathrm{~d} t$. El número inicial de saltos se supone igual a cero, es decir, $Q_{P, 0}=0$. En todo lo que sigue, se supondrá que $W_{P, t} \mathrm{y}$ $Q_{P, t}$ no están correlacionados entre sí.

\section{Activos de los consumidores}

El consumidor representativo tiene acceso a tres distintos activos en la economía: dinero, $M_{t}$; títulos de deuda pública, $B_{t}$; y títulos de capital, $k_{t}$. En consecuencia, la riqueza real, $a_{t}$, del individuo está dada por

$$
a_{t}=m_{t}+b_{t}+k_{t}
$$

1 Como siempre, o(h) significa que $o(h) / h \rightarrow 0$ cuando $h \rightarrow 0$. 
donde $m_{t}=M_{t} / P_{t}$ son los saldos monetarios reales y $b_{t}=B_{t} / P_{t}$ es la tenencia de bonos gubernamentales en términos reales. El consumidor obtiene satisfacción por el consumo del bien que produce la economía y por la tenencia de saldos reales, debido a sus servicios de liquidez. Suponemos que la función de utilidad esperada es del tipo von Neumann-Morgenstern. Específicamente, la función de utilidad total descontada al tiempo $t=0, V_{0}$, de un individuo representativo, competitivo y adverso al riesgo tiene la siguiente forma separable:

$$
V_{0}=\mathrm{E}\left[\int_{0}^{\infty}\left[\theta \log \left(c_{t}\right)+(1-\theta) \log \left(m_{t}\right)\right] e^{-\delta t} \mathrm{~d} t \mid \mathcal{F}_{0}\right],
$$

donde $\mathcal{F}_{0}$ es la información disponible en el tiempo $t=0$. Asimismo, la evolución de la acumulación de la riqueza real sigue la ecuación diferencial estocástica

$$
\mathrm{d} a_{t}=a_{t}\left[N_{m, t} \mathrm{~d} R_{m, t}+N_{b, t} \mathrm{~d} R_{b, t}+N_{k, t} \mathrm{~d} R_{k, t}\right]-c_{t}\left(1+\tau_{c}\right) \mathrm{d} t-\mathrm{d} \tau_{t},
$$

donde

$N_{j, t} \equiv \frac{j_{t}}{a_{t}}$ proporción del portafolio en el activo $j, j=m, b, k$;

$\mathrm{d} R_{j, t}=$ rendimiento después de impuestos sobre el activo $j, j=m, b, k$;

$\mathrm{d} \tau_{t}=$ impuestos sobre la riqueza; y

$\tau_{c}=$ impuesto sobre el consumo.

\section{Rendimiento de los activos}

A continuación se determinan los rendimientos de los activos. Se supone que las tasas nominales de rendimiento que pagan el dinero y los bonos son cero e $i$, respectivamente. El rendimiento estocástico por la tenencia de saldos reales al tiempo $t, \mathrm{~d} R_{m, t}$, es simplemente el cambio porcentual en el precio del dinero en términos de bienes. La aplicación del lema de Itô al cambio porcentual del inverso del nivel de precios, tomando (1) como el proceso subyacente, conduce a (véase el Apéndice $A$ ):

$$
\mathrm{d} R_{m, t}=P_{t} \mathrm{~d}\left(\frac{1}{P_{t}}\right)=r_{m} \mathrm{~d} t-\sigma_{P} \mathrm{~d} W_{P, t}+\left(\frac{1}{1+\nu_{P}}-1\right) \mathrm{d} Q_{P, t}
$$

donde $r_{m}=-\pi+\sigma_{P}^{2}$. El rendimiento estocástico por la tenencia de bonos se obtiene en forma similar como

$$
\mathrm{d} R_{b, t}=r_{b} \mathrm{~d} t-\sigma_{P} \mathrm{~d} W_{P, t}-\left(\frac{1}{1+\nu_{P}}-1\right) \mathrm{d} Q_{P, t}
$$

donde $r_{b}=i-\pi+\sigma_{P}^{2}$. Es importante observar que los rendimientos del dinero $\mathrm{y}$ de los bonos se ven afectados por la volatilidad y posibles saltos en el nivel 
general de precios. La tasa de rendimiento de las acciones después de impuestos será denotada mediante

$$
\mathrm{d} R_{k, t}=r_{k} \mathrm{~d} t+\sigma_{k} \mathrm{~d} W_{k, t}+\nu_{k} \mathrm{~d} Q_{k, t},
$$

donde los procesos $\mathrm{d} W_{k, t}$ y $\mathrm{d} Q_{k, t}$ tienen características similares a los procesos definidos en (1).

Además del impuesto $\tau_{c}$ que se paga por el consumo, el consumidor paga un impuesto sobre la riqueza de la forma

$$
\mathrm{d} \tau_{t}=a_{t} \bar{\tau} \mathrm{d} t+a_{t} \sigma_{\tau} \mathrm{d} W_{\tau, t}+a_{t} \nu_{\tau} \mathrm{d} Q_{\tau, t},
$$

donde $\bar{\tau}$ es la tasa impositiva media esperada sobre la riqueza real. Al igual que antes, $\mathrm{d} W_{\tau, t}$ y $\mathrm{d} Q_{\tau, t}$ comparten las mismas características que tienen los procesos de Wiener y de Poisson definidos en (1).

\section{Decisiones óptimas de los consumidores}

El objetivo del consumidor es elegir, en cada momento, el portafolio de activos y la cantidad de consumo que maximicen (5) sujeto a (6). Nótese que después de sustituir las expresiones (7)-(10) en la ecuación estocástica de acumulación de la riqueza, (6), ésta se transforma en

$$
\begin{aligned}
\frac{\mathrm{d} a_{t}}{a_{t}}= & {\left[N_{m, t} r_{m}+N_{b, t} r_{b}+N_{k, t} r_{k}-\frac{c_{t}\left(1+\tau_{c}\right)}{a_{t}}-\bar{\tau}\right] \mathrm{d} t } \\
& +\left[N_{k, t} \sigma_{k} \mathrm{~d} W_{k, t}-\left(N_{m, t}+N_{b, t}\right) \sigma_{P} \mathrm{~d} W_{P, t}-\sigma_{\tau} \mathrm{d} W_{\tau, t}\right] \\
& +\left[\left(N_{m, t}+N_{b, t}\right)\left(\frac{1}{1+\nu_{P}}-1\right) \mathrm{d} Q_{P, t}+N_{k, t} \nu_{k} \mathrm{~d} Q_{k, t}-\nu_{\tau} \mathrm{d} Q_{r, t}\right] .
\end{aligned}
$$

La solución del problema de maximización de utilidad total descontada sujeto a (11) y a la restricción de normalización

$$
N_{m, t}+N_{b, t}+N_{k, t}=1,
$$

están dadas por (véase el Apéndice B):

$$
\begin{gathered}
c_{t}=\frac{\delta \theta}{\left(1+\tau_{c}\right)} a_{t}, \\
0=\frac{\delta(1-\theta)}{N_{m, t}}+r_{m}-\left(N_{m, t}+N_{b, t}\right) \sigma_{P}^{2}+N_{k, t} \sigma_{P k}-\sigma_{P \tau} \\
-\frac{\lambda_{P} \nu_{P}}{1+\left(1-N_{m, t}-N_{b, t}\right) \nu_{P}}-\delta \phi, \\
0=\left[r_{b}-\left(N_{m, t}+N_{b, t}\right) \sigma_{P}^{2}+N_{k, t} \sigma_{P k}-\sigma_{P \tau}-\frac{\lambda_{P} \nu_{P}}{1+\left(1-N_{m, t}-N_{b, t}\right) \nu_{P}}\right]-\delta \phi
\end{gathered}
$$




$$
0=\left[r_{k}-N_{k, t} \sigma_{k}^{2}+\left(N_{m, t}+N_{b, t}\right) \sigma_{P k}+\sigma_{k \tau}+\frac{\lambda_{k} \nu_{k}}{1+\left(1-N_{m, t}-N_{b, t}\right) \nu_{k}}\right]-\delta \phi,
$$

donde $\phi$ es el multiplicador de Lagrange asociado a la restricción (12). Después de restar (14) de (15) encontramos la proporción óptima de la riqueza asignada a la tenencia de saldos reales:

$$
\widehat{N}_{m}=\frac{\delta(1-\theta)}{i}
$$

Asimismo, después de restar (15) de (16), se tiene que

$$
N_{k} B-A-\frac{\lambda_{P} \nu_{P}}{1+N_{k, t} \nu_{P}}-\frac{\lambda_{k} \nu_{k}}{1+N_{k, t} \nu_{k}}=0
$$

donde

$$
B \equiv \sigma_{P}^{2}+2 \sigma_{P k}+\sigma_{k}^{2}>0
$$

y

$$
A \equiv r_{k}-r_{b}+\sigma_{P}^{2}+\sigma_{P k}+\sigma_{P \tau}+\sigma_{k \tau} \text {. }
$$

Claramente, la ecuación (18) es cúbica y, por lo tanto, tiene al menos una solución real, la cual denotaremos por $\widehat{N}_{k}$. En particular, si suponemos que $\nu_{P}=\nu_{k}=0$, se tiene como única solución:

$$
\left.\widehat{N}_{k}\right|_{\nu_{P}=\nu_{k}=0}=\frac{A}{B}
$$

Si los parámetros $\nu_{P}$ y $\nu_{k}$ son de la misma magnitud y distintos de cero, entonces (18) se transforma en una ecuación cuadrática cuyas soluciones están dadas por:

$$
\left.\widehat{N}_{k}\right|_{\nu_{P}=\nu_{k}}=\frac{A \nu_{P}-B \pm \sqrt{\left(A \nu_{P}+B\right)^{2}+4 B \nu_{p}^{2}\left(\lambda_{p}+\lambda_{k}\right)}}{2 B \nu_{P}}
$$

Observe que el discriminante es positivo $y$, en consecuencia, ambas raíces son reales. Note también que en ningún caso se han impuesto restricciones para que las proporciones de la riqueza asignadas a la tenencia de activos sean estrictamente positivas y menores que la unidad. Por lo tanto, las ventas en corto de activos son permitidas en todo momento. Finalmente, el portafolio óptimo queda completamente determinado con $\widehat{N}_{b}$, el cual se obtiene a partir de (12) como

$$
\widehat{N}_{b}=1-\frac{\delta(1-\theta)}{i}-\widehat{N}_{k} .
$$




\section{Conclusiones}

Se desarolló un modelo que describe el comportamiento de un consumidorinversionista que toma decisiones de consumo y portafolio en un ambiente de riesgo e incertidumbre. Para esto, la estructura de la economía se mantuvo lo más simple posible. Sin embargo, el modelo se puede extender en varias direcciones, por ejemplo, incluir productos derivados, lo cual se llevará a cabo en el futuro.

\section{Apéndice A}

En este apéndice se establen sin demostración ${ }^{2}$ un par de resultados sobre la diferencial estocástica del cociente y la multiplicación de dos movimientos geométricos Brownianos. Dadas las ecuaciones diferenciales estocásticas, homogéneas y lineales,

$$
\mathrm{d} X_{t}=X_{t}\left(\mu_{X} \mathrm{~d} t+\sigma_{X} \mathrm{~d} W_{X}+\nu_{X} \mathrm{~d} Q_{X}\right)
$$

$\mathrm{y}$

$$
\mathrm{d} Y_{t}=Y_{t}\left(\mu_{Y} \mathrm{~d} t+\sigma_{Y} \mathrm{~d} W_{Y}+\nu_{Y} \mathrm{~d} Q_{Y}\right),
$$

donde $\mathrm{d} Q_{X}$ y $\mathrm{d} Q_{Y}$ son procesos de Poisson no correlacionados, $\mathrm{d} W_{X}, \mathrm{~d} W_{Y}$ son procesos de Wiener con $\operatorname{Cov}\left(\mathrm{d} W_{X}, \mathrm{~d} W_{Y}\right)=\rho_{X Y} \mathrm{~d} t$ y los procesos de Wiener son no correlacionados con los procesos de Poisson. En este caso, las diferenciales estocásticas del cociente $X_{t} / Y_{t}$ y del producto $X_{t} Y_{t}$ satisfacen, respectivamente,

$$
\begin{aligned}
\mathrm{d}\left(\frac{X_{t}}{Y_{t}}\right)= & \frac{X_{t}}{Y_{t}}\left[\left(\mu_{X}-\mu_{Y}+\sigma_{Y}^{2}-\sigma_{X Y}\right) \mathrm{d} t+\sigma_{X} \mathrm{~d} W_{X}-\sigma_{Y} \mathrm{~d} W_{Y}\right. \\
& \left.+\nu_{X} \mathrm{~d} Q_{X}+\left(\frac{1}{1+\nu_{Y}}-1\right) \mathrm{d} Q_{Y}\right]
\end{aligned}
$$

y

$\mathrm{d}\left(X_{t} Y_{t}\right)=X_{t} Y_{t}\left[\left(\mu_{X}+\mu_{Y}+\sigma_{X Y}\right) \mathrm{d} t+\sigma_{X} \mathrm{~d} W_{X}+\sigma_{Y} \mathrm{~d} W_{Y}+\nu_{X} \mathrm{~d} Q_{X}+\nu_{Y} \mathrm{~d} Q_{Y}\right]$.

\section{Apéndice B}

A continuación se determinan las condiciones de primer orden para una solución interior del problema de maximización de utilidad total del consumidor:

$$
\max _{c_{t}, N_{m, t}, N_{b, t}, N_{k, t}} \mathrm{E}_{0}\left\{\int_{0}^{\infty}\left[\theta \log \left(c_{t}\right)+(1-\theta) \log \left(N_{m, t} a_{t}\right)\right] e^{-\delta t} \mathrm{~d} t\right\},
$$

2 Véase Gihman and Skorohod (1972). 
sujeto a:

$$
\begin{aligned}
\frac{\mathrm{d} a_{t}}{a_{t}} & =\left[N_{m, t} r_{m}+N_{b, t} r_{b}+N_{k, t} r_{k}-\frac{c_{t}\left(1+\tau_{c}\right)}{a_{t}}-\tilde{\tau}\right] \\
& +\left[N_{k, t} \sigma_{k} \mathrm{~d} W_{k, t}-\left(N_{m, t}+N_{b, t}\right) \sigma_{P} \mathrm{~d} W_{P, t}-\sigma_{\tau} \mathrm{d} W_{\tau, t}\right] \\
& +\left[\left(N_{m, t}+N_{b, t}\right)\left(\frac{1}{1+\nu_{P}}-1\right) \mathrm{d} Q_{P, t}+N_{k, t} \nu_{k} \mathrm{~d} Q_{k, t}-\nu_{\tau} \mathrm{d} Q_{\tau, t}\right]
\end{aligned}
$$

y

$$
1-N_{m, t}-N_{b, t}-N_{k, t}=0
$$

donde $i, \pi, \tau_{c}, \tau_{y}, \bar{\tau}$, y las correspondientes varianzas y covarianzas son tomadas como dadas. La ecuación de Hamilton-Jacobi-Bellman (H-J-B) para el problema de control óptimo estocástico, planteado en (A.1)-(A.3), está dada por:

$$
\begin{aligned}
& \max _{c_{t}, N_{m, t}, N_{b, t}, N_{k, t}} H\left(c_{t}, N_{m, t}, N_{b, t}, N_{k, t} ; a_{t}\right) \equiv \\
& \max _{c_{t}, N_{m, t}, N_{b, t}, N_{k, t}}\left\{\theta \log \left(c_{t}\right)+(1-\theta) \log \left(N_{m, t} a_{t}\right)-\delta V\left(a_{t}\right)\right. \\
& +a_{t} V^{\prime}\left(a_{t}\right)\left[N_{m, t} r_{m}+N_{b, t} r_{b}+N_{k, t} r_{k}-\frac{c_{t}\left(1+\tau_{c}\right)}{a_{t}}-\bar{\tau}\right] \\
& +\frac{1}{2} a_{t}^{2} V^{\prime \prime}\left(a_{t}\right)\left[\left(N_{m, t}+N_{b, t}\right)^{2} \sigma_{P}^{2}+N_{k, t}^{2} \sigma_{k}^{2}+\sigma_{\tau}^{2}\right. \\
& \left.-2\left(N_{m, t}+N_{b, t}\right) N_{k, t} \sigma_{P k}+2\left(N_{m, t}+N_{b, t}\right) \sigma_{P \tau}-2 N_{k, t} \sigma_{k \tau}\right] \\
& +\lambda_{P}\left[V\left(a_{t}\left(1-\left(N_{m, t}+N_{b, t}\right) \frac{\nu_{P}}{1+\nu_{P}}\right)\right)-V\left(a_{t}\right)\right] \\
& +\lambda_{k}\left[V\left(a_{t}\left(1+N_{k, t} \nu_{k}\right)\right)-V\left(a_{t}\right)\right] \\
& \left.+\lambda_{\tau}\left[V\left(a_{t}\left(1-\nu_{\tau}\right)\right)-V\left(a_{t}\right)\right]+\phi\left(1-N_{m, t}-N_{b, t}-N_{k, t}\right)\right\}=0
\end{aligned}
$$

donde $\phi$ es el multiplicador de Lagrange asociado a la restricción de normalización (A.3), $V\left(a_{t}\right) e^{-\delta t}$ es la función de utilidad indirecta del agente, $\mathrm{y}$ $V^{\prime}\left(a_{t}\right) e^{-\delta t}$ es la variable de co-estado. La ecuación de H-J-B evaluada en el máximo se transforma en una ecuación diferencial ordinaria de segundo orden en $V\left(a_{t}\right)$. Se postula como candidato de solución a la función $V\left(a_{t}\right)=$ $\beta_{0}+\beta_{1} \log \left(a_{t}\right)$. Consecuentemente, 


$$
\begin{aligned}
& \max _{c_{t}, N_{m, t}, N_{b, t}, N_{k, t}} H\left(c_{t}, N_{m, t}, N_{b, t}, N_{k, t} ; a_{t}\right) \equiv \\
& \max _{c_{t}, N_{m, t}, N_{b, t}, N_{k, t}}\left\{\theta \log \left(c_{t}\right)+(1-\theta) \log \left(N_{m, t} a_{t}\right)-\delta\left[\beta_{0}+\beta_{1} \log \left(a_{t}\right)\right]\right. \\
& +\beta_{1}\left[N_{m, t} r_{m}+N_{b, t} r_{b}+N_{k, t} r_{k}-\frac{c_{t}\left(1+\tau_{c}\right)}{a_{t}}-\bar{\tau}\right] \\
& -\frac{1}{2} \beta_{1}\left[\left(N_{m, t}+N_{b, t}\right)^{2} \sigma_{P}^{2}+N_{k, t}^{2} \sigma_{k}^{2}+\sigma_{\tau}^{2}\right. \\
& \left.-2\left(N_{m, t}+N_{b, t}\right) N_{k, t} \sigma_{P k}+2\left(N_{m, t}+N_{b, t}\right) \sigma_{P \tau}-2 N_{k, t} \sigma_{k \tau}\right] \\
& +\beta_{1}\left[\lambda_{P} \log \left(1-\left(N_{m, t}+N_{b, t}\right) \frac{\nu_{P}}{1+\nu_{P}}\right)+\lambda_{k} \log \left(1+N_{k, t} \nu_{k}\right)+\lambda_{\tau} \log \left(1-\nu_{\tau}\right)\right] \\
& \left.+\phi\left(1-N_{m, t}-N_{b, t}-N_{k, t}\right)\right\}=0 .
\end{aligned}
$$

Por lo tanto, las condiciones necesarias de máximo están dadas por:

$$
0=\frac{\partial H}{\partial c_{t}}=\frac{\theta}{c_{t}}-\frac{\beta_{1}\left(1+\tau_{c}\right)}{a_{t}}
$$

$$
\begin{aligned}
& 0=\frac{\partial H}{\partial N_{m, t}}=\frac{1-\theta}{N_{m, t}} \\
& +\beta_{1}\left[r_{m, t}-\left(N_{m, t}+N_{b, t}\right) \sigma_{P}^{2}+N_{k, t} \sigma_{P k}-\sigma_{P \tau}-\frac{\lambda_{P} \nu_{P}}{1+\left(1-N_{m, t}-N_{b, t}\right) \nu_{P}}\right]-\phi ;
\end{aligned}
$$

$$
\begin{aligned}
0 & =\frac{\partial H}{\partial N_{b, t}} \\
& =\beta_{1}\left[r_{b}-\left(N_{m, t}+N_{b, t}\right) \sigma_{P}^{2}+N_{k, t} \sigma_{P k}-\sigma_{P+}-\frac{\lambda_{P} \nu_{P}}{1+\left(1-N_{m, t}-N_{b, t}\right) \nu_{P}}\right]-\phi ;
\end{aligned}
$$

$$
0=\frac{\partial H}{\partial N_{k, t}}=\beta_{1}\left[r_{k}-N_{k, \hat{\imath}} \sigma_{k}^{2}+\left(N_{m, t}+N_{b, t}\right) \sigma_{P k}+\sigma_{k \tau}+\frac{\lambda_{k} \nu_{k}}{1+\left(1-N_{m, t}-N_{b, t}\right) \nu_{k}}\right]-\phi ;
$$

$$
0=\frac{\partial H}{\partial \phi}=1-\left(N_{m, t}+N_{b, t}+N_{k, t}\right)
$$

Falta solamente determinar los coeficientes $\beta_{0}$ y $\beta_{1}$ definidos en $V\left(a_{t}\right)$. Después de sustituir los valores óptimos $\widehat{c}_{t}, \widehat{N}_{m}, \widehat{N}_{b}$ y $\widehat{N}_{k}$ en la condición H-J-B, se 
obtiene

$$
\begin{aligned}
0= & \left(1-\delta \beta_{1}\right) \log \left(a_{t}\right)+\theta \log (\theta)+(1-\theta) \log (1-\theta) \\
& -\theta \log \left[\beta_{1}\left(1+\tau_{c}\right)\right]-(1-\theta) \log \left[\beta_{1} i\left(1-\tau_{y}\right)\right]-\delta \beta_{0} \\
& +\beta_{1}\left[\widehat{N}_{m}\left(-\pi+\sigma_{P}^{2}\right)+\widehat{N}_{b} i\left(1-\tau_{y}\right)+\widehat{N}_{k} r_{k}-\frac{\widehat{c}_{t}\left(1+\tau_{c}\right)}{a_{t}}-\bar{\tau}\right] \\
& -\frac{1}{2} \beta_{1}\left[\left(\widehat{N}_{m}+\widehat{N}_{b}\right)^{2} \sigma_{P}^{2}+\widehat{N}_{k}^{2} \sigma_{k}^{2}+\sigma_{\tau}^{2}-2\left(\widehat{N}_{m}+\widehat{N}_{b}\right) \widehat{N}_{k} \sigma_{P k}\right. \\
& \left.+2\left(\widehat{N}_{m}+\widehat{N}_{b}\right) \sigma_{P \tau}-2 \widehat{N}_{k} \sigma_{k \tau}\right] \\
& +\beta_{1}\left[\lambda_{P} \log \left(1-\left(\widehat{N}_{m}+\widehat{N}_{b}\right) \frac{\nu_{P}}{1+\nu_{P}}\right)+\lambda_{k} \log \left(1+\widehat{N}_{k} \nu_{k}\right)+\lambda_{\tau} \log \left(1-\nu_{\tau}\right)\right]
\end{aligned}
$$

lo cual implica que $\beta_{1}=1 / \delta \mathrm{y}$

$$
\begin{aligned}
\beta_{0} & =\frac{\theta}{\delta} \log (\theta)+\frac{(1-\theta)}{\delta} \log (1-\theta)-\frac{\theta}{\delta} \log \left(\frac{1+\tau_{c}}{\delta}\right)-(1-\theta) \delta \log \left[\frac{i\left(1-\tau_{y}\right)}{\delta}\right] \\
& -\frac{\theta}{\delta}+\frac{1}{\delta^{2}}\left[\widehat{N}_{m}\left(-\pi+\sigma_{P}^{2}\right)+\widehat{N}_{b} i\left(1-\tau_{y}\right)+\widehat{N}_{k} r_{k}-\bar{\tau}\right] \\
& -\frac{1}{2} \frac{1}{\delta^{2}}\left[\left(\widehat{N}_{m}+\widehat{N}_{b}\right)^{2} \sigma_{P}^{2}+\widehat{N}_{k}^{2} \sigma_{k}^{2}+\sigma_{\tau}^{2}-2\left(\widehat{N}_{m}+\widehat{N}_{b}\right) \widehat{N}_{k} \sigma_{P k}\right. \\
& \left.+2\left(\widehat{N}_{m}+\widehat{N}_{b}\right) \sigma_{P \tau}-2 \widehat{N}_{k} \sigma_{k \tau}\right] \\
& +\frac{1}{\delta^{2}}\left[\lambda_{P} \log \left(1-\left(\widehat{N}_{m}+\widehat{N}_{b}\right) \frac{\nu_{P}}{1+\nu_{P}}\right)+\lambda_{k} \log \left(1+\widehat{N}_{k} \nu_{k}\right)+\lambda_{\tau} \log \left(1-\nu_{\tau}\right)\right] .
\end{aligned}
$$

\section{Bibliografía}

Gihman, I. I. and A. V. Skorohod (1972). Stochastic differential equations. Springer-Verlag, Berlin.

Venegas-Martínez, F. (2000). "On Consumption, Investment, and Risk. Economía Mexicana, Nueva Época, Vol. 9, No. 2, pp. 227-244.

Venegas-Martínez, F. (2005). Decisiones para la administración del riesgo macroeconómico. Denarius, Revista de Administración y Economía, No. 10, pp. 105-130. 